\title{
ON THE PANEL UNIT ROOT TESTS USING NONLINEAR INSTRUMENTAL VARIABLES*
}

\author{
Kyung So Im \\ University of Central Florida \\ M. Hashem Pesaran \\ University of Southern California and Cambridge University
}

October 25, 2003

\begin{abstract}
This paper re-examines the panel unit root tests proposed by Chang (2002). She establishes asymptotic independence of the t-statistics when integrable functions of lagged dependent variable are used as instruments even if the original series are cross sectionally dependent. From this rather remarkable result she claims that her non-linear instrumental variable (NIV) panel unit root test is valid under general error cross correlations for any $N$ (the cross section dimension) as $T$ (the time dimension of the panel) tends to infinity. We show that her claim is valid only if $N \ln T / \sqrt{T} \rightarrow 0$, as $N$ and $T \rightarrow \infty$, and this condition is unlikely to hold in practice, unless $N$ is very small. The favourable simulation results reported by Chang are largely due to her particular choice of the error correlation matrix, which results in weak cross section dependence. Also, the asymptotic independence property of the $t$-statistics disappears when Chang's modified instruments are used. Using a common factor model with a sizeable degree of cross section correlations, we are able to show that Chang's NIV panel unit root test suffers from gross size distortions, even when $N$ is small relative to $T$ (for example $N=5, T=100$ ).
\end{abstract}

JEL Classification: C12, C15, C22, C23.

Key Words: Non-linear Instrumental Variable (NIV) Panel unit root tests, Cross-section dependence, Finite sample properties.

${ }^{*}$ We would like to thank Michael Binder, George Kapetanios, and Junsoo Lee for helpful discussions and Mutita Akusuwan for computing the results reported in Table 5 of this paper. We are also grateful to Yoosoon Chang for providing us with her Gauss program. 


\section{Introduction}

Recently Chang (2002) has proposed a new panel unit root test based on the average of $t$-ratios computed from instrumental variable (IV) regressions using as instruments non-linear transformations of the lagged levels of the series under consideration. She shows that such non-linear IV $t$-ratios are asymptotically pair-wise uncorrelated as the time dimension of the panel, $T \rightarrow \infty$, even in the presence of cross section dependence, so long as the non-linear transformation used as the instrument is regularly integrable. This is a remarkable result and forms the basis of Chang's claim of having found a simple panel unit root test which is valid under quite general error cross correlations. In the abstract to her paper Chang states

"We show in the paper that such standardized sum of individual IV $t$-ratios has limit normal distribution so long as the panels have large individual time series observations and are asymptotically balanced in a very weak sense. We may have the number of cross-sectional units arbitrary small or large." (p. 261, emphasis added)

This paper re-examines this claim, particularly the part of the claim that relates to the possibility of the cross section dimension $(N)$, to be arbitrarily large. There are two issues. First, although the $t$-ratios are asymptotically independent as is shown in Chang (2002), asymptotic validity of panel unit root test which is based on sums of these IV $t$-ratios requires a much more restrictive condition to hold, namely

$$
\frac{N \ln T}{\sqrt{T}} \rightarrow 0, \text { as } N, T \rightarrow \infty
$$

This result follows because although the individual $t$-ratios in the panel unit root test are asymptotically independent, the variance of the average of the $t$ ratios tends to a non-zero limit as $N$ and $T \rightarrow \infty$, which depends on nuisance parameters. Therefore, in practice, Chang's NIV panel unit root test could at best be used only when $N$ is very small relative to $T$.

Assuming our rebuttal of Chang's claim is correct, we are left with the puzzle of explaining the very favourable Monte Carlo results reported by Chang (2002) for her proposed test. We first note that in her simulation experiments Chang in fact uses a "calibrated" version of the integrable function of lagged levels. In her theoretical discussion she focusses on

$$
F\left(y_{i, t-1}\right)=y_{i, t-1} e^{-c_{i}\left|y_{i, t-1}\right|},
$$

as an example, where $y_{i t}$ is the value of the $i^{\text {th }}$ cross section unit at time $t$, and $c_{i}$ is a fixed constant. But in her simulations she seems to have calibrated the choice of $c_{i}$ by experimenting with values that vary with the sample size, namely

$$
c_{i T_{i}}=\frac{K T_{i}^{-1 / 2}}{s_{i}}, s_{i}^{2}=\frac{1}{T_{i}} \sum_{t=1}^{T_{i}}\left(\Delta y_{i t}\right)^{2} \text {, }
$$


where $T_{i}$ is the time series dimension of the $i^{\text {th }}$ cross section, and $K$ is a fixed constant that she sets to 3 . Her footnote 7 suggests that this particular choice of $K$ has been arrived at after consideration of certain simulation results. Also, using $c_{i T_{i}}$ as defined in (2) results in a new instrument, to be denoted by $G\left(y_{i, t-1}\right)$, with different limit properties as compared to $F\left(y_{i, t-1}\right)$ in which $c_{i}$ does not vary with the sample size. Unlike $F\left(y_{i, t-1}\right)$, the modified (calibrated) instrument, $G\left(y_{i, t-1}\right)$, is asymptotically non-integrable, since with $K=3, c_{i T_{i}} \rightarrow 0$, as $T_{i} \rightarrow \infty$, and in the limit $G\left(y_{i, t-1}\right)$ and $y_{i, t-1}$ would behave very similarly.

More importantly, the degree of cross section dependence in Chang's simulations turns out to be very low, which is not sufficiently high to provide an informative check on the small sample validity of her proposed test, in general. This is investigated by Monte Carlo experiments using a residual common factor structure where we show that even if the calibrated form of $c_{i}$ favoured by Chang is used, the panel NIV test suffers from a significant degree of over rejection even for moderate values of $N$.

To deal with the problem of cross section dependence other panel unit root tests have also been proposed in the literature, for example, by Bai and $\mathrm{Ng}$ (2002), Moon and Perron (2003), and Phillips and Sul (2002), and Pesaran (2003). To provide a comparison with Chang's test we consider Pesaran's cross section augmented panel unit root test which is simple to implement and hence could be viewed as a reasonable alternative to NIV test. Small sample properties of the two tests are compared by Monte Carlo experiments.

The plan of the remainder of the paper is as follows: Next section sets out the model, briefly describes the NIV test and establishes the main theoretical results of the paper. Section 3 reports the simulation results. Section 4 concludes.

\section{NIV Unit Roots Tests}

Consider a sample of $N$ cross sections observed over $T$ time periods. We suppose that the stochastic process $y_{i t}$ is generated by the first-order autoregressive process: ${ }^{1}$

$$
y_{i t}=\phi_{i} y_{i, t-1}+\varepsilon_{i t}, \quad i=1, \ldots, N ; \quad t=1, \ldots, T,
$$

with given initial values, $y_{i 0}$. For each $i$, the error terms, $\varepsilon_{i t}$, are serially independent with $E\left(\varepsilon_{i t}\right)=0, \operatorname{Var}\left(\varepsilon_{i t}\right)=\sigma_{i}^{2}$ for all $t$. But for each $t, \varepsilon_{i t}$ and $\varepsilon_{j t}$ could be correlated such that $\operatorname{Cov}\left(\varepsilon_{i t}, \varepsilon_{j t}\right)=\sigma_{i j} \neq 0$, for all $i, j$ and $t$. Equation (4) can be expressed as

$$
\Delta y_{i t}=\beta_{i} y_{i, t-1}+\varepsilon_{i t}, \quad i=1, \ldots, N ; \quad t=1, \ldots, T .
$$

where $\beta_{i}=1-\phi_{i}$, and $\Delta y_{i t}=y_{i t}-y_{i, t-1}$. Under the null hypothesis of unit roots, we have

$$
H_{0}: \beta_{i}=0 \text {, for all } i \text {. }
$$

\footnotetext{
${ }^{1}$ Our analysis of Chang's test also applies to more complicated panel data models with intercepts, linear trends and serially correlated errors. But the simple model considered here suffices for illustrating our point. Chang also considers unbalanced panels where the time dimensions could differ over the cross section units.
} 
Under the alternative hypothesis,

$$
H_{A}: \beta_{i}<0 \text {, for some } i \text {. }
$$

The NIV procedure estimates $\beta_{i}$ using $F\left(y_{i, t-1}\right)$ as an instrument for $y_{i, t-1}$ in equation (5), where $F(\cdot)$ is an integrable function. Therefore, for the $i$-th time series, we have the NIV estimator of $\beta_{i}$ and its corresponding $t$-ratio:

$$
\hat{\beta}_{N I V, i}=\frac{\sum_{t=1}^{T} F\left(y_{i, t-1}\right) \Delta y_{i t}}{\sum_{t=1}^{T} F\left(y_{i, t-1}\right) y_{i, t-1}}, \quad t_{N I V, i}=\frac{\hat{\beta}_{N I V, i}}{s\left(\hat{\beta}_{N I V, i}\right)}=\frac{\sum_{t=1}^{T} F\left(y_{i, t-1}\right) \Delta y_{i t}}{\hat{\sigma}_{i} \sqrt{\sum_{t=1}^{T}\left[F\left(y_{i, t-1}\right)\right]^{2}}},
$$

where

$$
\hat{\sigma}_{i}^{2}=T^{-1} \sum_{t=1}^{T}\left(\Delta y_{i t}-\hat{\beta}_{N I V, i} y_{i, t-1}\right)^{2} .
$$

Park and Phillips $(1999,2001)$ showed that for each $i, t_{N I V, i}$ approaches to standard normal distribution under the null hypothesis as $T \rightarrow \infty$.

Chang's NIV unit root test statistic is obtained $a^{2}$

$$
Z_{N}=\frac{1}{\sqrt{N}} \sum_{i=1}^{N} t_{N I V, i}
$$

Given that $t_{N I V, i}$ is standard normal as $T$ tends to infinity, it is then easily seen that $Z_{N}$ also follows standard normal distribution for any $N$ if the series are cross-sectionally independent. Chang (2002) claims that this result holds even when the individual series are cross sectionally correlated.

Her claim is based on the following stochastic order results which hold for any positive definite matrix formed by $\sigma_{i j}{ }^{3}$

$$
\sum_{t=1}^{T} F\left(y_{i, t-1}\right) \varepsilon_{i t}=O_{p}\left(T^{1 / 4}\right), \quad \sum_{t=1}^{T}\left[F\left(y_{i, t-1}\right)\right]^{2}=O_{p}\left(T^{1 / 2}\right),
$$

and

$$
\sum_{t=1}^{T} F\left(y_{i, t-1}\right) F\left(y_{j, t-1}\right)=O_{p}(\ln T), \quad \text { for } i \neq j .
$$

From these it is easily deduced that

$$
\operatorname{Cov}\left(t_{N I V, i}, t_{N I V, j}\right)=O\left(\frac{\ln T}{\sqrt{T}}\right), \text { for all } i \neq j,
$$

which establishes that even in the presence of cross section dependence (with $\sigma_{i j} \neq 0$ ) the individual $N I V$ tratios, $t_{N I V, i}$, will be asymptotically independent as $T \rightarrow \infty$.

\footnotetext{
${ }^{2}$ See equation (16) in Chang (2002).

${ }^{3}$ See Kasahara and Kotani (1979) and Chang et al. (2001).
} 
This is a remarkable result which has led Chang to conclude that $Z_{N}$ will also tends to $N(0,1)$ as $T \rightarrow \infty$ for any $N{ }^{4}$ The proof offered by Chang (2002, p. 288) simply states that the asymptotic independence of $t_{N I V, i}$ and $t_{N I V, j}$ as $T \rightarrow \infty$ is sufficient for $Z_{N} \rightarrow_{d} N(0,1)$ for any $N$. No formal proof of is offered, however. Chang's theoretical result clearly holds if $N$ is fixed as $T \rightarrow \infty$. But, for $N$ and $T$ sufficiently large, it is not sufficient that $t_{N I V, i}$ 's are asymptotically uncorrelated. We also need to show that $\operatorname{Var}\left(Z_{N}\right) \rightarrow 1$ as $N$ and $T \rightarrow \infty$. But,

$$
\operatorname{Var}\left(Z_{N}\right)=\frac{1}{N} \sum_{i=1}^{N} \operatorname{Var}\left(t_{N I V, i}\right)+\frac{2}{N} \sum_{j=i+1}^{N} \sum_{i=1}^{N} \operatorname{Cov}\left(t_{N I V, i}, t_{N I V, j}\right) .
$$

The first term approaches unity as $T \rightarrow \infty$ regardless of the magnitude of $N$. But, the second term contains $N(N-1) / 2$ covariance terms, and although according to (12) each of these covariances are individually of order $\frac{\ln T}{\sqrt{T}}$, the sum of these covariances vanishes only if

$$
\frac{N \ln T}{\sqrt{T}} \rightarrow 0, \text { as both } N \text { and } T \rightarrow \infty .
$$

This places restrictions on the rate at which $N$ is allowed to rise relative to $T$ that are too restrictive to be relevant in practice where $N$ is larger than $T$, or are of the same orders of magnitude. ${ }^{5}$

\subsection{Choice of the Non-Linear Instrument}

The properties of the NIV approach also depends on the choice of the integrable function, $F\left(y_{i, t-1}\right)$, to be used as the instrument. As an important example, in the theoretical part of her paper Chang (2002) considers

$$
F\left(y_{i, t-1}\right)=y_{i, t-1} e^{-c_{i}\left|y_{i, t-1}\right|},
$$

where $c_{i}$ is a fixed constant. But in her simulations she allows $c_{i}$ to vary with $T$ and uses the modified function

$$
G\left(y_{i, t-1}\right)=y_{i, t-1} e^{-\frac{K\left|y_{i, t-1}\right|}{\hat{\sigma}_{i} \sqrt{T}}},
$$

where $\hat{\sigma}_{i}$ is a consistent estimator of $\sigma_{i}$ and $K$ is a fixed constant which she sets to 3 after some experimentation. ${ }^{6}$ As acknowledged by Chang (2002, p.277), her theory requires $c_{i}$ in (14) to be fixed, otherwise the asymptotic independence results in (10) and (11) would not hold. Using $G\left(y_{i, t-1}\right)$ in place of $F\left(y_{i, t-1}\right)$ which allows $c_{i}$ to depend on $T^{-1 / 2}$ impacts the asymptotics. The term $e^{-\frac{K\left|y_{i, t-1}\right|}{\hat{\sigma}_{i} \sqrt{T}}}$ in $G\left(y_{i, t-1}\right)$ as the factor discounting $y_{i, t-1}$ is bounded in probability and sums

\footnotetext{
${ }^{4}$ See Theorem 4.3 of Chang $(2002)$.

${ }^{5}$ Unfortunately, the problem cannot be resolved by resorting to a different type of normalization.

${ }^{6}$ See Change (2002, p.277) and footnote 7.
} 
involving $G\left(y_{i, t-1}\right)$ or $y_{i, t-1}$ will have the same orders asymptotically. Hence $t_{N I V, i}$ and $t_{N I V, j}$ obtained using the calibrated transformation, $G($.$) , will not$ be asymptotically uncorrelated if the underlying series are cross-sectionally correlated.

Figure 1 illustrates the effect of using $G\left(y_{i, t-1}\right)$ instead of $F\left(y_{i, t-1}\right)$ on the distribution of $t_{i, N I V}$ for $T=100,1000,10000$. The chart at the top of the figure relates to $F\left(y_{i, t-1}\right)$ and the one at the bottom to $G\left(y_{i, t-1}\right)$. When they are compared to the standard normal density, it becomes clear that the density of the NIV $t$-ratio based on the integrable function, $F\left(y_{i, t-1}\right)$, converges rapidly to the standard normal density with $T$ rising, as the theory predicts. But the density of the NIV $t$-ratio obtained using the non-integrable function, $G\left(y_{i, t-1}\right)$, remains flatter than standard normal density even at $T=10,000$.

A natural question now is, "Why is $G\left(y_{i, t-1}\right)$ used in place of $F\left(y_{i, t-1}\right)$ ?" In standard panel unit root tests, under cross section independence, where the panel unit root is obtained as an average of the $t$-ratios of individual time series, it is more important to ensure that the individual $t$-ratios are centered appropriately under the null hypothesis. Shape of the density of the statistic is of secondary importance. Incorrect centering of the statistics becomes increasingly more problematic as $N$ increases, and could result in substantial size distortions as $N$ grows. However, the distribution of the average statistic quickly converges to normal, even if the density of individual $t$-ratios are quite different from that of normal density. Therefore, appropriate centering of the average statistic is far more important than the density of the underlying individual $t$-ratios. As is seen in Figure 1, the mean of the $t$-ratio obtained from the calibrated instrument, $G\left(y_{i, t-1}\right)$, is centered at zero more accurately, and is stable for different sample sizes, while the density of $t_{i, N I V}$ obtained using $F\left(y_{i, t-1}\right)$ is more skewed to the right, particularly when $T$ is relatively small. Therefore, it is not surprising that using $G\left(y_{i, t-1}\right)$ as an instrument performs better than $F\left(y_{i, t-1}\right)$ in testing for unit root in panels. In fact, using $F\left(y_{i, t-1}\right)$ yields NIV panel unit root tests that are grossly under-sized, with size being zero or very close to zero, and shows little power. In the next section, where we consider the small sample properties of the NIV test, we follow Chang and use $G\left(y_{i, t-1}\right)$ as the instrument. This also ensures comparability of our simulation results with those reported by Chang.

\section{Finite Sample Properties: Monte Carlo Ex- periments}

As was discussed in the previous section, NIV test is not robust to the presence of cross-sectional correlations, and therefore we may expect the size of NIV test to be different from the nominal size in the case where the errors are crosssectionally dependent. However, the simulation results reported in Chang (2002) which are based on the modified instrumental variables, $G\left(y_{i, t-1}\right)$ exhibit little size distortions. This seems to contradict our argument. But it turns out that the favourable Monte Carlo results reported by Chang are largely due to the 
particular way that she generates correlations across errors.

She draws the errors from an $N$ dimensional multivariate normal distribution with a covariance matrix whose elements are randomly drawn, such that some cross sectional units are positively correlated and others are negatively correlated. The average of the correlations in Chang's covariance matrix depends on $N$, and tend to be quite small. For example, the average of the covariance terms is about 0.07 when $N=10 .^{7}$ It is therefore also important to check the validity of Chang's test in cases where the cross section dependence of the errors is relatively large. Therefore, in addition to Chang's covariance structure we shall also consider a simple one-factor residual specification with an average cross correlations of around $0.2{ }^{8}$ Otherwise, for comparability we shall follow Chang's simulation design closely. We consider the sample size combinations: $N$ $=5,15,25,50,100$, and $T=25,50,100$, and use the following data generating (DGP) process

$$
\begin{aligned}
& x_{i t}=\phi_{i} x_{i, t-1}+u_{i t}, \quad u_{i t}=\rho_{i} u_{i, t-1}+\varepsilon_{i t}, \quad i=1, \ldots N ; \quad t=1, \ldots, T \\
& \left\{\begin{array}{l}
z_{i t}=\alpha_{i}, \quad \text { for the time series with no time trend. } \\
z_{i t}=\alpha_{i}+\beta_{i} t, \quad \text { for the time series with linear time trend. }
\end{array}\right. \\
& y_{i t}=x_{i t}+z_{i t}, \quad i=1, \ldots N ; \quad t=1, \ldots, T .
\end{aligned}
$$

The error terms follow $\mathrm{AR}(1)$ processes with the coefficient, $\rho_{i}$, drawn from the uniform distribution $[0.2,0.4]$, and the serial correlations are controlled by augmenting the NIV regressions with $\Delta y_{i, t-1}$. Under the null hypothesis, $\phi_{i}=1$ for all $i$, and under the alternative hypothesis, $\phi_{i} \sim i i d U[0.8,1.0]$. We set $\alpha_{i}=20$ and $\beta_{i}=0.3$, for all $i$, and use 10,000 replications for each of the experiments.

The cross section dependence is introduced in two ways: (i) the covariance matrix used in Chang, which we denote by $\Omega_{c}$, and (ii) by generating $\varepsilon_{i t}$ using the following single-factor model

$$
\varepsilon_{i t}=\gamma_{i} f_{t}+v_{i t}
$$

where $f_{t}$ and $v_{i t}$ are generated as $i i d N(0,1)$, and $\gamma_{i} \sim i i d U[-1,3]$. This specification generates the average cross section correlation coefficient of around 0.18. ${ }^{9}$ Under Chang's covariance specification, we generate an $N \times 1$ vector of independent standard normal random numbers for each time period first, then pre-multiply them by $\Omega_{c}^{1 / 2}$ to generate $\varepsilon_{i t}, i=1,2, \ldots, N$.

\footnotetext{
${ }^{7}$ See Chang (2002, p. 276) for further details.

${ }^{8}$ This set up has also been utilized by Bai and Ng (2002), Moon and Perron (2003), and Phillips and Sul (2002), and Pesaran (2003) in their Monte Carlo experiments.

${ }^{9}$ For given values of $\gamma_{i}$, the cross section correlation coefficient of $\varepsilon_{i t}$ and $\varepsilon_{j t}$ is given by

$$
\frac{\gamma_{i} \gamma_{j}}{\sqrt{\left(1+\gamma_{i}^{2}\right)\left(1+\gamma_{j}^{2}\right)}}, \text { for } i \neq j \text {. }
$$

Due to the independence of $\gamma_{i}$ and $\gamma_{j}$ the average of these correlation coefficients across $i$ and $j$ is given by $\left[E\left(\gamma_{i} / \sqrt{1+\gamma_{i}^{2}}\right)\right]^{2}$, where $\gamma_{i} \sim i i d U[-1,3]$. Using stochastic simulations the average correlation coefficient is around 0.18 which is much smaller than 0.50 , the value that obtains in the homogeneous case where $\gamma_{i}=1$.
} 


\subsection{Small Sample Results for NIV Test}

Tables 1-2 report the results for Chang's error covariance matrix, $\Omega_{c}$. Table 1 gives the results for models with an intercept only, and Table 2 for models with a linear trend. The results in Table 1 confirm Chang's findings for this case. These tables also give the corresponding results for Im, Pesaran, and Shin (2003, IPS) test which also show satisfactory size properties despite the cross section dependence. Note, however, that the IPS size results reported in Table 1 are closer to the nominal $5 \%$ value than in the simulations reported by Chang. This discrepancy seems to be primarily due to the demeaning procedure used by Chang. When she computed IPS statistic, Chang demeaned the observations on the whole series first, and then run Augmented Dickey-Fuller regression without an intercept. This should be fine when one works with a single time series, but could distort the mean adjustment needed for properly centering the IPS statistic in the case of panels where $T$ is small relative to $N .^{10}$

The power of Chang's test critically depends on whether the model contains a linear trend. It performs better than the IPS test when the model contains an intercept only, but shows little power when a linear trend is included. This is puzzling and could be due to the calibration of the instrument parameters $(K / \sqrt{T}$, with $K=3)$ in the former case. Chang does not report Monte Carlo results for the models with a linear trend.

The results for the single-factor covariance structure are given in Tables 3 and 4, and clearly show that both tests (NIV and IPS) are grossly over-sized, with the size increasing with $N$, as predicted by the arguments in the previous section. The size distortions could not have been seen in the earlier experiments simply because the cross correlations generated under Chang's design had been very weak.

\subsection{A Comparison of CADF and NIV Tests}

It is important that panel unit root tests are robust to high as well as low degrees of cross section dependence. As noted in the introduction a number of such tests have been recently proposed in the literature. In Table 5 we compare the size and power of the NIV test with that of the cross section augmented test recently proposed by Pesaran (2003). This test is based on Dickey-Fuller regressions further augmented with the cross section averages $\bar{y}_{t-1}$, and $\Delta \bar{y}_{t}$ :

$$
\Delta y_{i t}=a_{i}+b_{i} y_{i, t-1}+c_{i} \bar{y}_{t-1}+d_{i} \Delta \bar{y}_{t}+e_{i t}, i=1,2, \ldots, N
$$

In the case of serially correlated errors the above cross sectionally augmented Dickey-Fuller (CADF) regressions need to be further augmented with the lagged changes $\Delta y_{i, t-1}, \Delta y_{i, t-2, . . ;} \Delta \bar{y}_{t-1}, \Delta \bar{y}_{t-2}, \ldots$ The cross section averages act as asymptotically perfect proxies for the common factor generating the cross correlations. The test statistic proposed by Pesaran (2003) is defined by the simple

\footnotetext{
${ }^{10}$ We are grateful to Chang for providing us with her Gauss program that enabled us to identify the source of the discrepancy between the IPS results that she reports and the ones that we obtain.
} 
average of the CADF statistics, given by the OLS $t$-ratios of $b_{i}, i=1,2, \ldots, N$. Appropriate critical values for this test is provided in Tables 3a-3c of Pesaran (2003). A truncated version of the test, referred to as CIPS*, is also proposed which is shown to have better size properties when $T$ is very small, say less than 15. The results in Table 5 once again clearly show the substantial size distortion of the NIV test for moderate degrees of error cross correlations. It also shows that in the same setting, the size of the CIPS* test is close to its nominal value even for very small sample sizes, and its power rises quite rapidly with increases in $N$ and $T$. See Pesaran (2003) for a more detailed discussion of the CADF test and its small sample performance in more general set ups, including when the errors are serially correlated.

\section{Concluding Remarks}

We review the Chang's NIV panel unit root test; its asymptotics as well as the small sample properties. Unlike the author's claim, NIV test is not robust to the presence of cross-sectional correlations. The test is valid when the data are cross-sectionally independent, and seems to have satisfactory power properties when the model contains an intercept only. However, when time series are trended, power of NIV test declines significantly. This could be due to the choice of the calibrated non-linear instruments used by Chang (2002). In cases where cross section dependence is not too weak it is advisable that other panel unit root tests proposed in the literature are considered.

\section{References}

[1] Bai, J. and S. Ng, (2002), "A Panic Attack on Unit Roots and Cointegration", Department of Economics, Boston College, Unpublished Manuscript.

[2] Chang, Y., (2002), "Nonlinear IV Unit Root Tests in Panels with CrossSectional Dependence", Journal of Econometrics 110, 261-292.

[3] Chang, Y., J. Park, and P.C.B. Phillips, (2001), "Nonlinear Econometric Models with Cointegrated and Deterministically Trending Regressors," Econometrics Journal, 4, 1-36.

[4] Im, K., Pesaran, H., and Y. Shin, (2003), "Testing for Unit Roots in Heterogeneous Panels," Journal of Econometrics 115, 53-74.

[5] Kasahara, Y., and S. Kotani, (1979), "On the Limit Process for a Class of Additive Functionals of Recurrent Diffusion Processes," Zeitschrift fuer Wahrscheinlichkeitstheorie verw Gebiete 49, 133-153.

[6] Moon, H.R., and B. Perron, (2003), "Testing for a Unit Root in Panels with Dynamic Factors", Journal of Econometrics (forthcoming). 
[7] Park, J., and P.C.B. Phillips, (1999), "Asymptotics for Nonlinear Transformation of Integrated Time Series," Econometric Theory 15, 269-298.

[8] Park, J., and P.C.B. Phillips, (2001), "Nonlinear Regression with Integrated Time Series," Econometrica 69, 117-162.

[9] Pesaran, M. Hashem, (2003) "A Simple Panel Unit Root Test in the Presence of Cross Section Dependence", http://www.econ.cam.ac.uk/faculty/pesaran/panelcadf.pdf

[10] Phillips, P.C.B. and D. Sul, (2002), "Dynamic Panel Estimation and Homogeneity Testing Under Cross Section Dependence", Cowles Foundation Discussion Paper No. 1362, Yale University. 


\section{Figure 1 \\ Density of NIV $t$-statistics}

Using Integrable Function, $F($.$) , as Instrument$

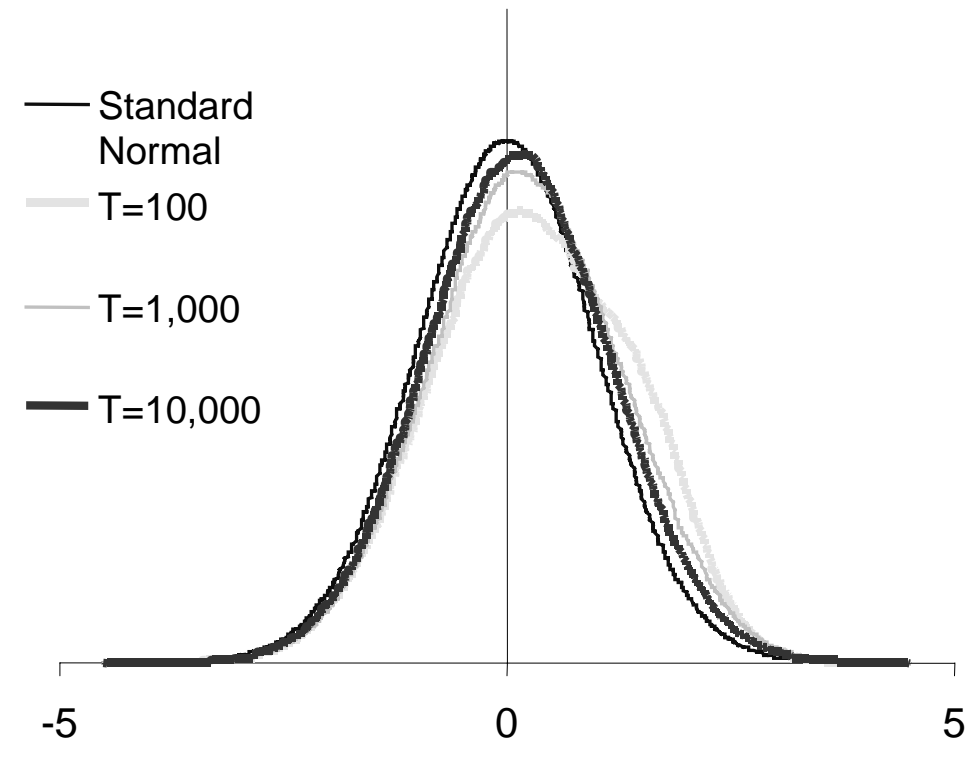

Using Chang's Modified Instrument, G(.)

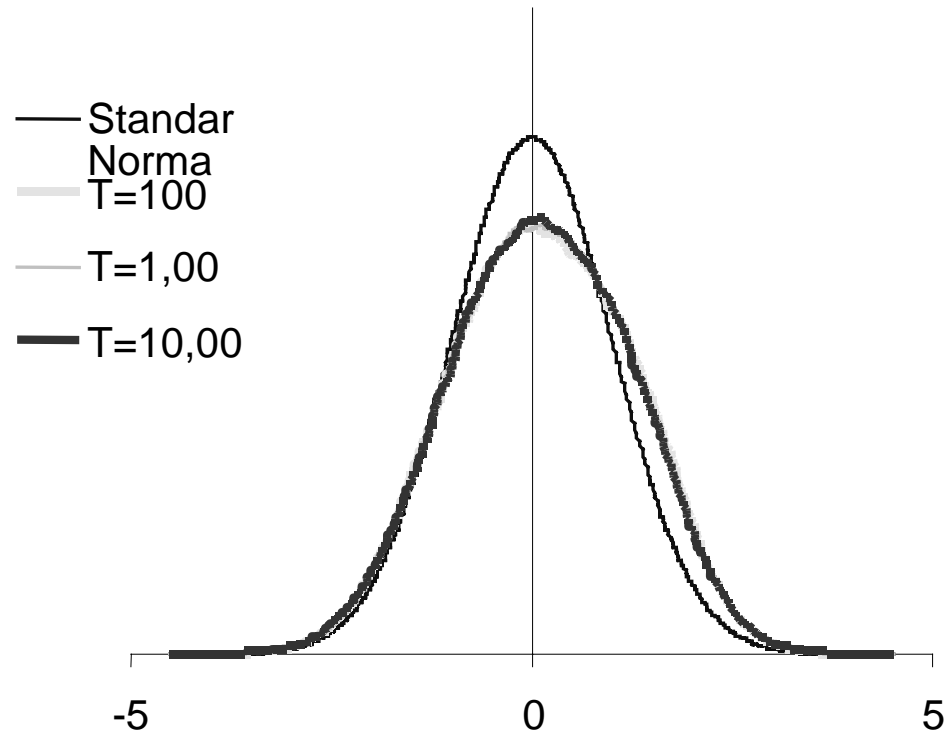


Table 1: CHANG'S CROSS CORRELATIONS: With an Intercept Only

\begin{tabular}{|c|c|c|c|c|c|c|c|}
\hline & & \multicolumn{2}{|c|}{$T=25$} & \multicolumn{2}{c|}{$T=50$} & \multicolumn{2}{c|}{$T=100$} \\
\hline & & SIZE & POWER & SIZE & POWER & SIZE & POWER \\
\hline$N=5$ & & & & & & & \\
\hline & IPS & 0.070 & 0.188 & 0.063 & 0.241 & 0.066 & 0.715 \\
\hline & NIV & 0.055 & 0.410 & 0.063 & 0.458 & 0.065 & 0.925 \\
\hline$N=15$ & & & & & & & \\
\hline & IPS & 0.059 & 0.314 & 0.060 & 0.834 & 0.060 & 1.000 \\
\hline & NIV & 0.050 & 0.692 & 0.049 & 0.994 & 0.053 & 1.000 \\
\hline$N=25$ & & & & & & & \\
\hline & IPS & 0.070 & 0.557 & 0.062 & 0.942 & 0.066 & 1.000 \\
\hline & NIV & 0.046 & 0.942 & 0.054 & 1.000 & 0.056 & 1.000 \\
\hline$N=50$ & & & & & & & \\
\hline & IPS & 0.067 & 0.707 & 0.067 & 1.000 & 0.065 & 1.000 \\
\hline & NIV & 0.042 & 0.992 & 0.044 & 1.000 & 0.050 & 1.000 \\
\hline$N=100$ & & & & & & & \\
\hline & IPS & 0.065 & 0.934 & 0.061 & 1.000 & 0.060 & 1.000 \\
\hline & NIV & 0.035 & 1.000 & 0.032 & 1.000 & 0.036 & 1.000 \\
\hline
\end{tabular}

Table 2: CHANG'S CROSS CORRELATIONS: With a Linear Trend

\begin{tabular}{|c|c|c|c|c|c|c|c|}
\hline & & \multicolumn{2}{|c|}{$T=25$} & \multicolumn{2}{c|}{$T=50$} & \multicolumn{2}{c|}{$T=100$} \\
\hline & & SIZE & POWER & SIZE & POWER & SIZE & POWER \\
\hline$N=5$ & & & & & & & \\
\hline & IPS & 0.072 & 0.108 & 0.071 & 0.174 & 0.070 & 0.323 \\
\hline & NIV & 0.049 & 0.047 & 0.067 & 0.090 & 0.086 & 0.157 \\
\hline$N=15$ & & & & & & & \\
\hline & IPS & 0.067 & 0.115 & 0.064 & 0.541 & 0.067 & 0.895 \\
\hline & NIV & 0.041 & 0.041 & 0.058 & 0.152 & 0.074 & 0.419 \\
\hline$N=25$ & & & & & & & \\
\hline & IPS & 0.071 & 0.173 & 0.071 & 0.526 & 0.074 & 0.999 \\
\hline & NIV & 0.043 & 0.043 & 0.060 & 0.141 & 0.082 & 0.889 \\
\hline$N=50$ & & & & & & & \\
\hline & IPS & 0.077 & 0.213 & 0.069 & 0.771 & 0.072 & 1.000 \\
\hline & NIV & 0.035 & 0.037 & 0.059 & 0.197 & 0.072 & 0.965 \\
\hline$N=100$ & & & & & & & \\
\hline & IPS & 0.069 & 0.351 & 0.064 & 0.962 & 0.070 & 1.000 \\
\hline & NIV & 0.034 & 0.035 & 0.050 & 0.282 & 0.071 & 0.999 \\
\hline
\end{tabular}


Table 3: CROSS SECTION CORRELATIONS GENERATED BY A COMMON FACTOR MODEL: With an Intercept Only

\begin{tabular}{|c|c|c|c|c|c|c|c|}
\hline & & \multicolumn{2}{|c|}{ T=25 } & \multicolumn{2}{c|}{ T=50 } & \multicolumn{2}{c|}{$T=100$} \\
\hline & & SIZE & POWER & SIZE & POWER & SIZE & POWER \\
\hline$N=5$ & & & & & & & \\
\hline & IPS & 0.098 & 0.204 & 0.097 & 0.336 & 0.095 & 0.654 \\
\hline & NIV & 0.083 & 0.412 & 0.091 & 0.591 & 0.094 & 0.893 \\
\hline$N=15$ & & & & & & & \\
\hline & IPS & 0.180 & 0.370 & 0.181 & 0.733 & 0.180 & 0.998 \\
\hline & NIV & 0.157 & 0.591 & 0.164 & 0.922 & 0.163 & 1 \\
\hline$N=25$ & & & & & & & \\
\hline & IPS & 0.234 & 0.526 & 0.238 & 0.672 & 0.239 & 0.955 \\
\hline & NIV & 0.195 & 0.787 & 0.214 & 0.887 & 0.218 & 0.995 \\
\hline$N=50$ & & & & & & & \\
\hline & IPS & 0.308 & 0.625 & 0.312 & 0.851 & 0.326 & 0.992 \\
\hline & NIV & 0.271 & 0.833 & 0.279 & 0.967 & 0.293 & 1 \\
\hline$N=100$ & & & & & & & \\
\hline & IPS & 0.378 & 0.683 & 0.390 & 0.900 & 0.384 & 0.997 \\
\hline & NIV & 0.332 & 0.863 & 0.342 & 0.985 & 0.353 & 1 \\
\hline
\end{tabular}

Table 4: CROSS SECTION CORRELATIONS GENERATED BY A COMMON FACTOR MODEL: With a Linear Trend

\begin{tabular}{|c|c|c|c|c|c|c|c|}
\hline & & \multicolumn{2}{|c|}{ T=25 } & \multicolumn{2}{c|}{ T=50 } & \multicolumn{2}{c|}{ T=100 } \\
\hline & & SIZE & POWER & SIZE & POWER & SIZE & POWER \\
\hline$N=5$ & & & & & & & \\
\hline & IPS & 0.106 & 0.139 & 0.100 & 0.217 & 0.114 & 0.382 \\
\hline & NIV & 0.087 & 0.072 & 0.095 & 0.12 & 0.132 & 0.180 \\
\hline$N=15$ & & & & & & & \\
\hline & IPS & 0.188 & 0.242 & 0.190 & 0.477 & 0.185 & 0.968 \\
\hline & NIV & 0.164 & 0.155 & 0.191 & 0.277 & 0.203 & 0.809 \\
\hline$N=25$ & & & & & & & \\
\hline & IPS & 0.24 & 0.330 & 0.248 & 0.435 & 0.236 & 0.794 \\
\hline & NIV & 0.205 & 0.211 & 0.228 & 0.277 & 0.247 & 0.548 \\
\hline$N=50$ & & & & & & & \\
\hline & IPS & 0.305 & 0.410 & 0.32 & 0.625 & 0.312 & 0.932 \\
\hline & NIV & 0.276 & 0.277 & 0.311 & 0.400 & 0.315 & 0.727 \\
\hline$N=100$ & & & & & & & \\
\hline & IPS & 0.366 & 0.469 & 0.371 & 0.695 & 0.387 & 0.953 \\
\hline & NIV & 0.326 & 0.336 & 0.353 & 0.473 & 0.373 & 0.789 \\
\hline
\end{tabular}


Table 5: The Size and Power of Panel Unit Root tests: No Serial Correlation, High Cross Section Dependence, Intercept Case ${ }^{1}$

\begin{tabular}{|c|c|c|c|c|c|c|c|}
\hline & & \multicolumn{2}{|c|}{ T=25 } & \multicolumn{2}{c|}{ T=50 } & \multicolumn{2}{c|}{ T=100 } \\
\hline & & SIZE & POWER & SIZE & POWER & SIZE & POWER \\
\hline$N=5$ & & & & & & & \\
\hline & CIPS $^{*}$ & 0.054 & 0.087 & 0.049 & 0.233 & 0.073 & 0.681 \\
\hline & NIV & 0.138 & 0.392 & 0.131 & 0.679 & 0.150 & 0.963 \\
\hline$N=15$ & & & & & & & \\
\hline & CIPS $^{*}$ & 0.055 & 0.142 & 0.059 & 0.507 & 0.040 & 0.991 \\
\hline & NIV & 0.259 & 0.642 & 0.223 & 0.870 & 0.226 & 0.998 \\
\hline$N=25$ & & & & & & & \\
\hline & CIPS $^{*}$ & 0.050 & 0.141 & 0.042 & 0.658 & 0.054 & 1.00 \\
\hline & NIV & 0.295 & 0.718 & 0.28 & 0.934 & 0.246 & 1.00 \\
\hline$N=50$ & & & & & & & \\
\hline & CIPS $^{*}$ & 0.043 & 0.218 & 0.046 & 0.849 & 0.051 & 1.00 \\
\hline & NIV & 0.379 & 0.762 & 0.381 & 0.960 & 0.378 & 1.00 \\
\hline$N=100$ & & & & & & & \\
\hline & CIPS $^{*}$ & 0.037 & 0.242 & 0.061 & 0.963 & 0.044 & 1.00 \\
\hline & NIV & 0.485 & 0.808 & 0.420 & 0.976 & 0.418 & 1.00 \\
\hline & & & & & & & \\
\hline
\end{tabular}

Notes: ${ }^{1}$ The results in this Table are based on the Monte Carlo design used in Pesaran (2003). The underlying data is generated by $y_{i t}=\left(1-\phi_{i}\right) \mu_{i}+\phi_{i} y_{i, t-1}+u_{i t}, i=1,2, . ., N, t=-51,-50, \ldots, T$, and $u_{i t}=\gamma_{i} f_{t}+\varepsilon_{i t}$ where $\gamma_{i} \sim \operatorname{iid} U[-1,3], \mu_{i}$ and $f_{t} \sim \operatorname{iidN}(0,1)$, and $\varepsilon_{i t} \sim \operatorname{iidN}\left(0, \sigma_{i}^{2}\right)$ with $\sigma_{i}^{2} \sim \operatorname{iid} U[0.5,1.5]$. The Size ( under the null $\phi_{i}=1$ ) of the CIPS* test is computed at the $5 \%$ nominal level, based on cross section augmented Dickey-Fuller (CADF) regressions:

$\Delta y_{i t}=a_{i}+b_{i} y_{i, t-1}+c_{i} \bar{y}_{t-1}+d_{i 0} \Delta \bar{y}_{t}+u_{i t}, i=1,2, . ., N, t=1,2, \ldots, T$ where $\bar{y}_{t}=N^{-1} \sum_{i=1}^{N} y_{i t}$.

The power of the test is computed at the $5 \%$ nominal level based on the same regression under the heterogeneous alternative of $\phi_{i} \sim \operatorname{iid} U[0.85,0.95] . C^{*} I P S^{*}=N^{-1} \sum_{i=1}^{N} C A D F^{*}{ }_{i}$, where $C A D F^{*}{ }_{i}$ is the OLS t-ratio of $b_{i}$ truncated to lie in the interval [-6.19,2.16]. Chang's NIV statistic is computed based on Chang (2002) under the model with a non-zero mean and no linear trend (cf. Chang (2002)'s Eq.(18)) and the time lag of order one. The size of the Chang's NIV test is computed at the one-sided 5\% nominal level, using the standard normal distribution. The simulation results reported in this table are based on 1,000 replications. 